\title{
Hierarchical Hidden Markov Model for Finger Language Recognition
}

\author{
Jae-Hong Kwon ${ }^{\text {a }}$, Hong-Jun Joo ${ }^{\text {, }}$, Tae-Young Kim ${ }^{\text {, }}$ * \\ ${ }^{\text {a,b,c }}$ Chung-Ang University, Heuksuk-Dong, Dongjak-Ku 156-756, Seoul, South Korea \\ *Corresponding Author: kimty@cau.ac.kr
}

\begin{abstract}
The finger language is the part of the sign language, which is a language system that expresses vowels and consonants with hand gestures. Korean finger language has 31 gestures and each of them needs a lot of learning models for accurate recognition. If there exist mass learning models, it spends a lot of time to search. So a real-time awareness system concentrates on how to reduce search spaces. For solving these problems, this paper suggest a hierarchy HMM structure that reduces the exploration space effectively without decreasing recognition rate. The Korean finger language is divided into 3 categories according to the direction of a wrist, and a model can be searched within these categories. Pre-classification can discern a similar finger Korean language. And it makes a search space to be managed effectively. Therefore the proposed method can be applied on the real-time recognition system. Experimental results demonstrate that the proposed method can reduce the time about three times than general HMM recognition method.
\end{abstract}

Keywords: hand recognition, hierarchical model, hidden Markov model.

\section{Introduction}

A study on the $\mathrm{HCI}$ (Human-Computer interaction) internationally has been going positively. For designing system more natural and intellectual between human and computer, there becomes important issues on visual language like sign language(SL) or finger language(FL). There are lots of trying on the research about recognition system of SL and FL. Manjula ${ }^{(1)}$ accomplished 84\% average of recognition rate on 14 gestures of SL at recognition system with applying artificial neural network ${ }^{(1)}$. In case of Yamaguchi ${ }^{(2)}$, there has been studied on a system that results $82 \%$ average of recognition rate using a date-glove, extracting 16 of Japan SL features from system and image which recognizes 34 FL among 46 FL. Seun-Ki Min and Hee-Deok Yang ${ }^{(3)}$, has researched on study using the data-glove and method that recognizes SL and FL based on image ${ }^{(4 \sim 5)}$. There are 2 kinds of main problems to be solved on following recognition system of SL and FL. The first problem is reducing mis-recognition rate on similar gestures. The second problem, for real-time recognition, is the need of efficient management on searching DB model. For solving these problems, this paper suggests Korean FL HMM recognition system which is applied pre-classification, which gets data of hands on 3D using Leap Motion ${ }^{(6)}$ without preprocessing or wearing equipment

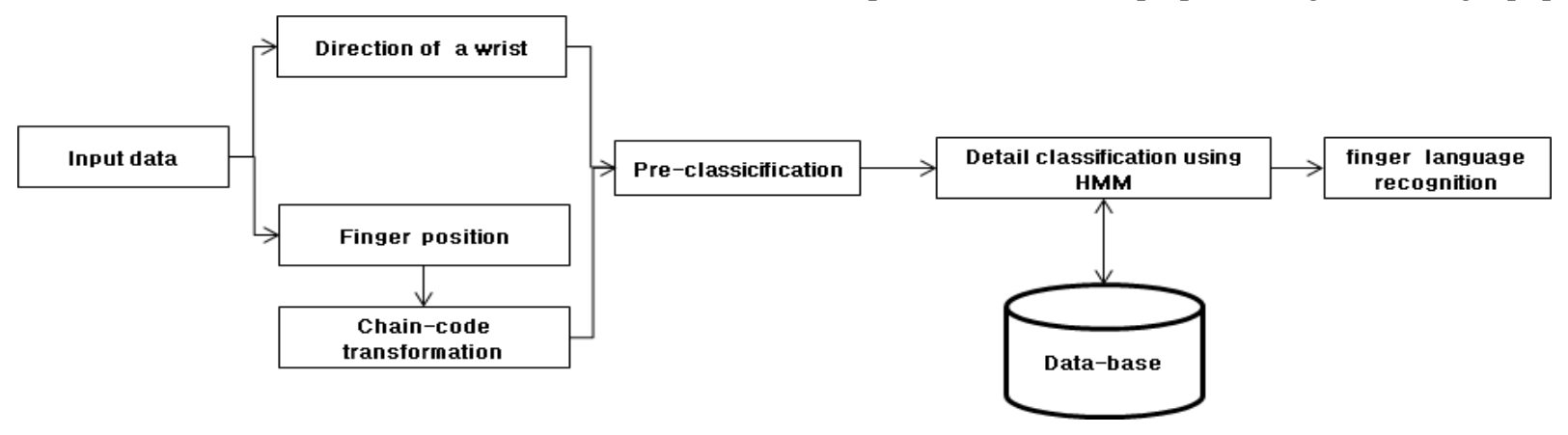

Fig. 1. The proposed Korean finger language recognition system. 
(Fig. 1).

\section{Hierarchical Korean finger language recognition system}

\subsection{Traget gestures}

Korean is composed with basic consonant, complex consonant, basic vowel and complex vowel total 40 kinds of phoneme. As Tenchijin Keyboard is available in mobile with basic consonant and vowel of Korean, although this paper is trying to recognize 31 kinds of FL, for accurate recognition and efficient manage of system resource, basic consonants and vowels are applied on Tenchijin Keyboard that recognizes 11 FL gestures (Fig 2).

\section{$2.2 \quad$ Feature extraction}

According to the recent study ${ }^{(7)}$, the feature in gesture recognition is categorized by location of object, angle and velocity. The result that each of the feature value is applied to gesture recognition system shows recognition rates as $46 \%$ of extracted location of a object, $87 \%$ of angle of object and $32 \%$ of velocity of a object. As a result, the angle of a object is the most efficient feature point on the gesture recognition system. Therefore, in this paper, as based on following study, fingers angle is used as features for gesture recognition system. Leap motion provides a variety of data for the hands. In this paper, use the position of the fingers,

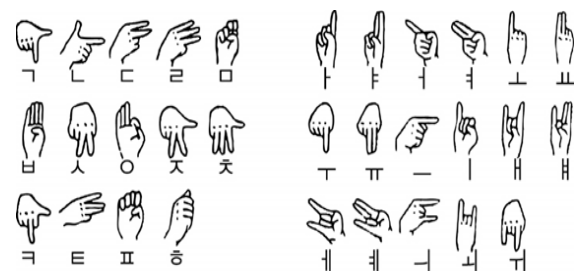

(a)

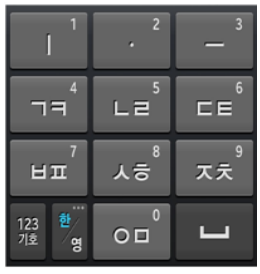

(b)

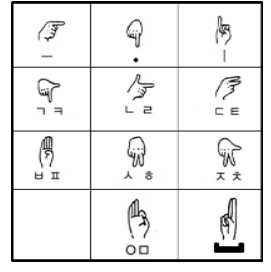

(c)
Fig. 2. (a) Korean finger language (b) Tenchijin keyboard, (c) Tenchijin applied to the keyboard of Korean finger language. and hand torques data. Calculating the differentials of the finger position, and generates a chain code of 27 directions. And the chain code has a total of 28 ranges by adding state of fingers Non-tracking. Acquired chain-code build 5 dimensional vector as below.

$$
\mathrm{V}=(\text { C_thumb, C_index finger, ... })
$$

where C_thumb means the chain code of a thumb. Also means the rest fingers is the same. The direction of a wrist is categorized by Pitch, Roll and Yaw at Leap Motion. Data which is used on proposed gesture recognition system is Pitch value, which is the angle between projected $\mathrm{Z}$ axis and Y-Z plane.

\subsection{Using HMM recognition}

In simpler Markov models, it does not accurately model complex situations. Through many years of research of the Markov model, $\mathrm{HMM}^{(8)}$ is developed. HMM is a double-probability-model that is used to recognize a pattern of handwriting, voice, gesture and etc. ,which solves various problems. Observation sequence, expressed with object pattern, is the way that analyzes as string and makes to learn as probability model, and calculates an observation probability for input signal. To train a model creates a probability model which has maximum probability for observation sequence.

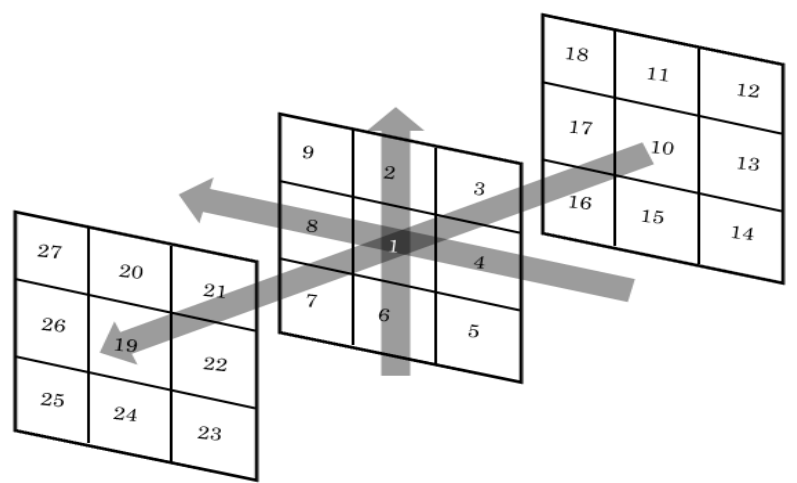

Fig. 3. 27-direction of 3 dimensional chain-code. 


\subsection{Pre-classification}

Category of proposed Korean FL is divided into three classes by the direction of wrist as Fig. 4. Category which is supposed to be searched is selected by the Pitch value of a wrist and reference models. Korean FL defines wrist direction as 3 categories. There is two reasons doing pre-classification. First, general HMM searches the whole references for an input, but proposed method searches part of all models and can reduce search spaces. Second, among the Korean FL gestures, there exist characters that are hard to recognize because of similar gestures. For example, 'ᄃ-E' and '人-む' are possible to consider as same gestures except the direction of wrist. By categorizing DB models, the pre-classification is able to reduce mis-recognition on similar gestures, and consequently recognition rate is increased.

\subsection{Gesture spotting}

Because it is not able to know the start/end point at real-time, spotting of gestures is applied as following condition $^{(7)}$. When hands are moving $(\mathrm{H}-\mathrm{M})$, it regards as starting of a gesture and saves an input data as shown in Fig. 5. And grabbing hand $(\mathrm{GH})$ is defined as end of the gesture. If GH state keeps during a bit of a frame, input data are compared with DB models, and the state becomes idle(NH)

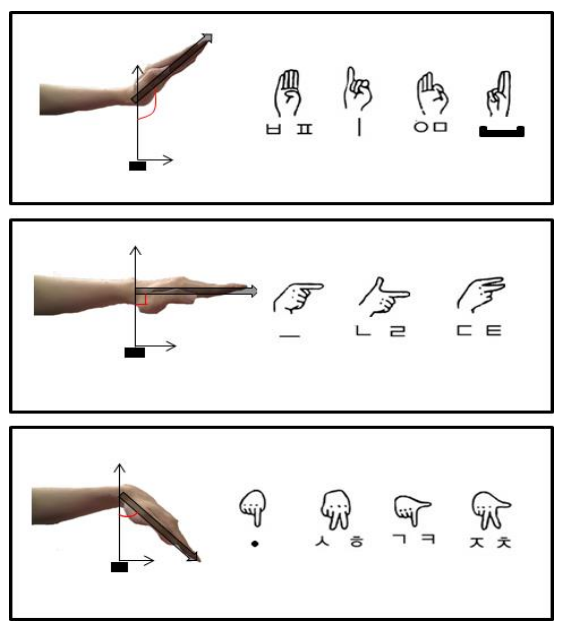

Fig. 4. Korean finger language classified by the direction of a wrist.

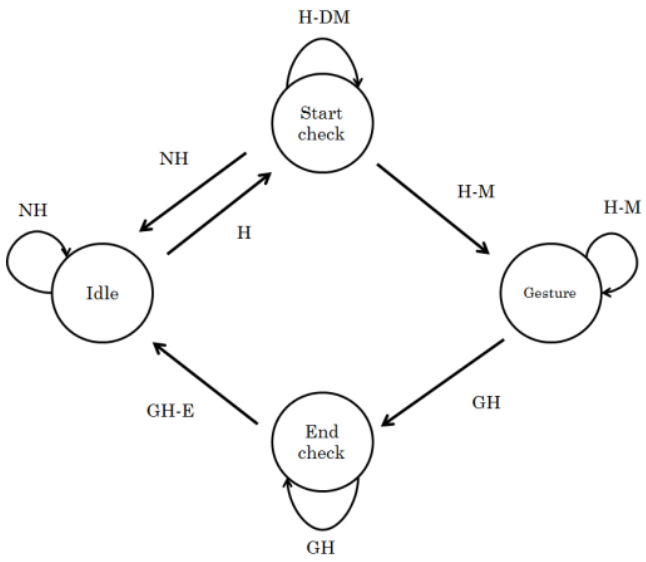

\begin{tabular}{|l|l|l|}
\hline Symbol & $\begin{array}{l}\text { Results of hand } \\
\text { extraction }\end{array}$ & $\begin{array}{l}\text { Result of the hand } \\
\text { movement. }\end{array}$ \\
\hline H & Hand exists. & movement. \\
\hline NH & No Hand exists. & None \\
\hline H-DM & Hand exists. & Non - movement. \\
\hline H-M & Hand exists. & movement. \\
\hline GH & Hand exists. & Non - movement. \\
\hline GH-E & Hand exists. & Non - movement. \\
\hline
\end{tabular}

Fig. 5. States for gesture spotting.

\section{Experiment}

Table 1 shows recognition rate according to proposed method(H-HMM), general HMM, and each state numbers. According to the Table 1, there is a lots of differences between general HMM and H-HMM. In case of general HMM recognition method, there is no pre-classification and input data is similar to every model therefore mis-recognition rate is increased. There is a lot of difference at recognition rate on similar gesture (“ᄂ,己” and "ᄀ, Э“, "—“ and “ । " or Additional stroke .. etc) without the direction of a wrist and less difference at recognition rate on discrimination gestures as “ $\bigcirc, \square ”$, “ㅂ, 프". Besides, study as changing state number in Korean FL recognition system using HMM accomplished the highest recognition rate on fine states. If state numbers are too small at HMM structure, recognition rate is declined. Although expanding state numbers, it shows a little difference on recognition rate and increasing matching time. Table 2 shows searching process speed on one DB model for each of methods. Proposed method takes 2 3 times lost than general HMM recognition method because of using pre-classification. Also matching time within DB is increased as increasing state number on each method. 
Table 1. Recognition experimental results.

\begin{tabular}{|c|c|c|c|c|c|c|c|c|c|c|c|c|}
\hline Ratio(\%) & - & $\begin{array}{c}\text { Additional } \\
\text { stroke }\end{array}$ & 1 & $\neg, \neg$ & ᄂ,己 & ᄃ, E & 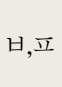 & 入, $\overline{0}$ & 下, 之 & ○,口 & Space & Average \\
\hline H-HMM-3S & 81.67 & 71.67 & 75.00 & 83.33 & 91.67 & 90.00 & 81.67 & 76.67 & 88.33 & 80.00 & 85.00 & 82.27 \\
\hline H-HMM-4S & 86.67 & 81.67 & 81.67 & 91.67 & 95.00 & 95.00 & 90.00 & 85.00 & 95.00 & 85.00 & 90.00 & 88.79 \\
\hline H-HMM-5S & 86.67 & 83.33 & 85.00 & 91.67 & 96.67 & 95.00 & 91.67 & 86.67 & 96.67 & 88.33 & 93.33 & 90.45 \\
\hline HMM-3S & 75.00 & 58.33 & 70.00 & 60.00 & 35.00 & 78.33 & 80.00 & 60.00 & 83.33 & 75.00 & 75.00 & 68.18 \\
\hline HMM-4S & 80.00 & 70.00 & 76.67 & 70.00 & 76.67 & 83.33 & 88.33 & 70.00 & 88.33 & 81.67 & 81.67 & 78.79 \\
\hline HMM-5S & 80.00 & 73.33 & 78.33 & 71.67 & 78.33 & 78.33 & 88.33 & 73.33 & 88.33 & 85.00 & 83.33 & 79.85 \\
\hline HMM-6S & 83.33 & 66.67 & 81.67 & 71.67 & 70.00 & 60.00 & 81.67 & 65.00 & 85.00 & 83.33 & 80.00 & 75.30 \\
\hline
\end{tabular}

\section{Conclusions}

In this paper, we suggest a HMM recognition system to recognize 11 kinds of finger gestures using two information that is wrist direction and finger position. The main goal of this paper is to reduce mis-recognition rate on HMM gesture recognition system and to match efficiently by the categorized direction for Korean FL gestures. To show the superiority of the proposed method, comparative experiments are conducted with general HMM recognition system. In optimal state, the recognition rate of proposed method is $90 \%$ on average and it is $10 \%$ higher than general HMM system. The proposed method showed two to three times faster than the general HMM. If we conduct a gesture spotting more naturally in real-time, it is expected to build a robust recognition system for the applications.

\section{Acknowledgment}

This work was supported by the National Research Foundation of Korea grant (MOE(NRF2014S1A5B6037633) and Basic Science Research Program through the National Research Foundation of Korea(NRF) funded by the Ministry of Education, Science and Technology(2013-009166).

\section{References}

(1) Manjula, Waldron, Soowon Kim, "Isolated ASL Sign Recognition System for Deaf persons", Rehabilitation Engineering, IEEE Transactions on, Vol. 3, pp. 261-271, 1995.

(2) Yamaguchi, "Japanese sign language recognition system using information infrastructure", International Joint Conference of the Fourth IEEE International Conference on Fuzzy Systems and
Table 2. DB model matching speed.

\begin{tabular}{|c|c|}
\hline method & second \\
\hline H-HMM-3S & 0.0075 \\
\hline H-HMM-4S & 0.0076 \\
\hline H-HMM-5S & 0.0077 \\
\hline H-HMM-6S & 0.0078 \\
\hline \hline HMM-3S & 0.0192 \\
\hline HMM-4S & 0.0194 \\
\hline HMM-5S & 0.0195 \\
\hline HMM-6S & 0.0197 \\
\hline
\end{tabular}

The Second International Fuzzy Engineering Symposium, Vol. 5, pp. 65-66, 1995.

(3) Seun-Ki Min, Sang-Yeok Oh, Gyor-Yeong Kim, Tae-Hyun Yoon, Chun-Gyu Lim, Kee-Chul-Jung, Yunli Lee, "Optimize Data Glove-based System for Korean Finger Spelling Recognition”, Korea Computer Congress, Vol. 34, pp. 237-241, 2007.

(4) Hee-Deok Yang, Seong-Whan Lee, "Automatic Spotting of Sign and Fingerspelling for Continuous Sign Language Recognition", Korean Institute of Information scientists and Engineers, Vol. 38, pp 102-107, 2011.

(5) Min-Ji Kang, Eun-Sook Choi, Young-Sun Sohn, "The Study on Dynamic Images Processing for Finger Languages", Korean Institute of Intelligent Systems, Vol 14, pp. 184-189, 2004.

(6) Frank Weichert, "Analysis of the Accuracy and Robustness of the Leap Motion Controller", Sensors, Vol. 13, pp. 6380-6393, 2013.

(7) Lawrence Rabiner, "A tutorial on hidden Markov models and selected applications in speech recognition", Proceedings of the IEEE, Vol 77, pp. 257-286, 1989.

(8) Ho-Sub Yoon, Jung-Soh, Young-Lae Bae, Hyun-Seung Yang, "Hand gesture recognition using combined features of location, angle and velocity", Pattern recognition, Vol. 34, pp. 1491-1501, 2001. 